\title{
Determination of Risk Factors Associated With Walking Disorders After Anterior Cruciate Ligament Surgery With Hamstring Graft : A Controlled Multicenter Study \\ Marine GAIGNON ${ }^{1}$, Jean MAZEAS ${ }^{1}$, Maude TRAULLÉ ${ }^{1}$, Amaury VANDEBROUCK ${ }^{2}$, Pascal DUFFIET ${ }^{2}$, Louis RATTE ${ }^{2}$, Florian FORELLI ${ }^{1}$
}

${ }^{1}$ Researcher Physical Therapist, OrthoLab, 85 route de Domont, 95330 Domont, France

${ }^{2}$ Knee Orthopaedic Surgeon, Clinic of Domont, 85 route de Domont, 95330 Domont, France

\section{ABSTRACT}

Today, rupture of the anterior cruciate ligament is the most common heavy injury in athletes, but it can also occur in sedentary subjects. Its treatment, always adapted to the lifestyle, age and will of the patient, often remains surgical. However, despite its frequency and universality, there are still walking disorders following the operation. These disorders sometimes persist for several months, or even several years after surgery. The present study is therefore interested in determining the risk factors linked to walking disorders following reconstruction of the anterior cruciate ligament.

The objective of this study is to determine what risk factors associated with walking disorders are after reconstruction of the anterior cruciate ligament by hamstring graft. This study focused on factors that may exist at 3 and 6 months postoperatively.

Two populations participated in this study, a healthy population and a population having benefited from reconstruction of the anterior cruciate ligament. The operated test group is divided into two subgroups, one with a postoperative period of 3 months and the second at 6 months. All subjects were subjected to the same protocol, their gait on the treadmill was analyzed and evaluated with an Optogait ${ }^{\circledR}$ analysis system. A univariate analysis was carried out first. Then, a multivariate analysis by adjustment method was carried out in order to eliminate the potential confounding factors.

The comparison of the results between the populations in the univariate analysis shows an absence of significant results however there are trends.

The statistical results of the multivariate analysis showed interactions in the two subgroups of the operated population.

In fact, it turns out that at 3 months there is a tendency to worse gait disorders in subjects with a high $\mathrm{BMI}$ reflecting overweight, when an associated surgical procedure has taken place on the meniscus, in subjects over 35 years of age and males. On the other hand, at 6 months, the tendency to worsening is visible only in the strata represented by subjects with a BMI corresponding to overweight, as well as in subjects having benefited from a meniscal suture associated with ACL reconstruction. .

The results of the present study show that there are certain factors which tend to increase the risk of these walking disorders after reconstruction of the anterior cruciate ligament by hamstring graft, these factors are different depending on the time postoperative. 
Keywords : Anterior cruciate ligament, gait disorder, gait analysis, risk factors.

\section{INTRODUCTION}

Anterior cruciate ligament (ACL) tear is the most frequent serious ligament injury with $65 \%$ of heavy injuries in sports (1). Indeed, it occurs in $20 \%$ of cases when the knee undergoes an altercation during physical activity. (2)

These ruptures of the anterior cruciate ligament are increasingly treated surgically in order to avoid the early appearance of associated pathologies such as meniscal pathologies or the early onset of osteoarthritis. Indeed, the risk of developing meniscal lesions or chronic knee instability is $20 \%$. More than $50 \%$ of patients with an ACL deficiency show radiologic evidence of osteoarthritis of the knee ten years after their injury (3-5) .

Today, we know that reconstruction can reduce the consequences of osteoarthritis as well as anteroposterior and antero-external instability (6) .

Increasingly, reconstruction takes place in relatively short time after injury.

Some authors consider that, as early as six months after an ACL rupture, without operative intervention, one can observe symptoms of degeneration of the menisci.

Thus, they recommend reconstruction within a year of injury to avoid these complications (7).

In a sports population, the percentage of surgical reconstruction is considered to be $76.6 \%$ (8). For example, France recorded 41,000 anterior cruciate ligament reconstruction operations in 2012, which represents a real concern for public health as well as a significant economic cost.

Often, despite ACL reconstruction, asymmetries persist in both lower limbs. These asymmetries are responsible for walking disorders which appear from the first postoperative month up to 6 months or even a few years after the surgery (9-16).

It is not uncommon for associated surgery on the meniscus to worsen these walking disorders $(12,13)$. A fortiori, we imagine that these walking alterations affect not only the function of the musculoskeletal system and the kinematics of the operated limb, but also represent a danger for the walking parameters of the non-operated limb (17). These walking disorders, as they are described in the literature, can be responsible in the long term for the appearance of other disorders and injuries.

In order to be able to offer the best possible rehabilitation to patients, it seems important to determine whether there are risk factors for gait disturbances following $A C L$ reconstruction.

Thus, we may wonder what the risk factors for post-ACLR by hamstring graft walking disorders at 3 months and 6 months are.

\section{METHOD}

Participants 
The present study was carried out using two groups, an operated test group and a non-operated control group. A total of 90 subjects were added to the study. The operated test group consists of 65 patients (age $31.53 \pm 12.11-\mathrm{BMI}=22.6 \pm 3.42$ ) and the control group of 25 patients (age $33.19 \pm 11.06-\mathrm{BMI}=$ $25,09 \pm 3.64)$. Were included in the test group only patients over 18 years of age, having benefited from reconstruction of the cruciate ligament prior to DIDT and able to walk without walking assistance at the postoperative period of 3 months or 6 months. Furthermore, the patients had to have a dry and noninflammatory knee without hematoma and pain less than 2 on Visual Analog Scale (VAS). Were excluded from the two groups all the subjects who presented, on both lower limbs, neurological, dental and/or orthopedic disorders with the exception of the surgical procedure obviously. Likewise, subjects who had consumed psychotropic substances before the test were excluded.

In the control group, the inclusion criteria were the same as in the test group. The subject was also to present a gait without visible lameness and without walking aid.

Protocol

The protocol implemented was identical for all subjects participating in the study.

Each subject performed the walking test on the Elite 5000 treadmill from NordicTrack * on which the OpToGait *device (Version 1.6.4.0, Microgate", Bolzano, Italy) presented by Microgate is installed. The latter device was connected to a Dell * computer which has a 256GB SSD, a 1TB hard drive, an intel core i7 * processor, 8GB of RAM, and Windows 10 .

OptoGait *is an optical detection system. It works with a transmitter bar and a receiver bar; it has an acquisition frequency of $1000 \mathrm{~Hz}$ and a spatial precision of $1 \mathrm{~cm}$.

Each of these bars contains 96 leds. These LEDs make it possible, for example, to measure the flight and contact time during the execution of a series of jumps, with an accuracy of 1/1000 of a second. The OpToGait device is validated for research because it has an ICC greater than 95\% (18) which allowed us to obtain reliable data for the measurements obtained.

During the test, each subject was allowed a 10-minute adaptation time (3) on the treadmill. This adaptation time made it possible to reproduce a walking pattern that was as natural as possible. During this adaptation period, the treadmill's speed was set to $4.0 \mathrm{~km} / \mathrm{h}$.

Once the elapsed adaptation, the treadmill is stopped, and gait analysis is started on OptoGait software. The selected test is already offered by the OptoGait system. The test is the following "Treadmill Walking $4 \mathrm{~km} / \mathrm{h}$ or $2.5 \mathrm{mph}$ ». The latter imposes a walking speed of $4 \mathrm{~km} / \mathrm{h}$.

Table I : Demographic table of the control and control groups (mean \pm standard deviation )

\begin{tabular}{|c|c|c|c|}
\hline & A group of witnesses & Test group & $\rho$-value \\
\hline & $(n=25)$ & $(n=61)$ & \\
\hline & mean \pm standard deviation & mean \pm standard deviation & \\
\hline Age (a) & $31.53 \pm 12.11$ & $33.19 \pm 11.06$ & NS \\
\hline
\end{tabular}




\begin{tabular}{llll} 
BMI & $22.66 \pm 3.42$ & $25.09 \pm 3.64$ & 0.004 \\
Gender (M / F) & $11 / 14$ & $38 / 23$ & NS \\
Operated side (R / L) & $\varnothing$ & $35 / 26$ & $/$ \\
\hline $\begin{array}{l}\text { a : years - kg : kilogram - m : meter - BMl : body mass index kg / } \mathrm{m}^{2}-\mathrm{M} / \mathrm{F}: \text { male / female - D / G : right / left - } \varnothing \square: \text { no value - / : value } \\
\text { cannot be calculated }\end{array}$
\end{tabular}

\section{Statistical analysis}

Statistical analyzes were performed using $R^{\circledR}$ software ( $R$ Studio, Version 1.2.5033 (C) 20092019 RStudio , Inc - 250 Northern Ave, Boston, MA 02210) after exporting the data to Excel ${ }^{\circledR}$ as well as to the using the software Epi Info тм I CDC.

Interference statistics have been carried out. The confidence level is preset such that C $=95 \%$ and the significance level is $\alpha=0.05$.

The study includes 86 subjects, it is therefore necessary to perform a normality test in order to account for the distribution of populations.

In order to compare the demographic data of each patient, we therefore used the Shapiro test on each of the populations of the sample.

The study of the variances is carried out on each of the populations of the sample thanks to the Chi2 test which is chosen for the qualitative values as well as Mann and Whitney (Wilcoxon-MannWhitney) for quantitative values.

Following the study of the descriptive variables, it is a question of establishing the ORb (gross Odd Ratio) to objectify the walking disorders.

The goal is to evaluate the risk of having walking disorders when one has undergone an anterior cruciate ligament repair.

First, the calculation of the ORa (adjusted Odd Ratio) was carried out by a varied uni analysis. Determining the ORa helps us shed light on the risk of having trouble walking. The unvaried analysis was carried out using the exact Fischer test which determines the threshold of significance.

For each stratum, in order to identify certain factors independently of other potential confounding factors, a multivariate analysis was carried out secondly using the Cochran-Mantel-Haenszel adjustment method.

\section{RESULTS}

Table II: Table listing the ORbs of the different groups among the test and control populations.

TDM

not (\%) $\varnothing \square T D M$

GOLD

$95 \% \mathrm{Cl}$

$\rho \square(b)$ 


\begin{tabular}{|c|c|c|c|c|c|c|c|}
\hline A group of witnesses & 4 & (16) & 21 & (84) & 0.22 & [0.0688-0.732] & 0.0132 \\
\hline 3 month test group & 16 & (50) & 16 & (50) & 5.25 & {$[1,4682-18,7728]$} & 0.0114 \\
\hline 6 month test group & 12 & (41.3) & 17 & (58.7) & 3.7 & [1.0102-13.5951] & NS \\
\hline Test group (global) & 28 & $(46)$ & 33 & (54) & 4.45 & [1.3662-14.5247] & 0.0132 \\
\hline $\begin{array}{l}\text { (b) } \mathrm{NS}=\text { non-significant diffe } \\
\mathrm{CT}=\text { trouble walking } \\
\mathrm{OR}=\text { Odd Ratio } \\
\mathrm{Cl}=\text { confidence interval } \\
\varnothing \square=\text { absence }\end{array}$ & scher & Fischer ex & & & & & \\
\hline
\end{tabular}

Table III : Results obtained for the test group at the operating time of 3 months.

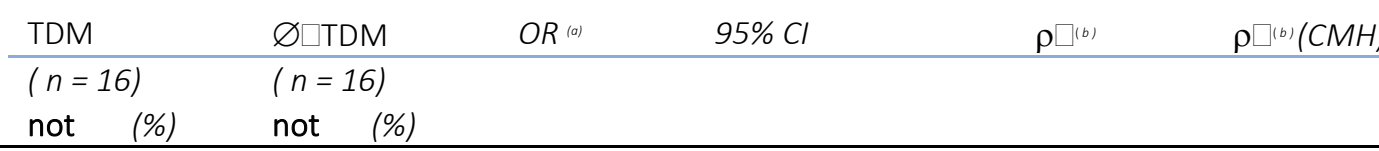

Age (c)

$\begin{array}{lllllllll}18-25 \text { years } & 4 & (12.5) & 6 & (18.75) & 0.67 & {[0.15-2.8209]} & \text { NS } & \text { NS } \\ 25-35 \text { years } & 2 & (6.25) & 4 & (12.5) & 0.5 & {[0.0799-3.1276]} & \text { NS } & \text { NS } \\ >35 \text { years old } & 10 & (31.25) & 6 & (18.75) & 1.67 & {[0.4888-5.6829]} & \text { NS } & 0.0124\end{array}$

BMI (c)

$\begin{array}{lllllllll}18.5-24.99 & 8 & (25) & 7 & (21.9) & 1.14 & {[0.3346-3.9041]} & \text { NS } & 0.0347 \\ >24.99 & 9 & (28.1) & 7 & (21.9) & 1.29 & {[0.3847-4.2969]} & \text { NS } & 0.0261\end{array}$

\begin{tabular}{|c|c|c|c|c|c|c|}
\hline \multicolumn{7}{|c|}{ ACL reconstruction by DIDT ligamentoplasty ${ }^{(c)}$} \\
\hline Isolated & 8 & (25) & 12 & $(37.5)$ & 0.67 & {$[0.15$} \\
\hline Associated meniscectomy & 2 & (6.25) & 1 & (3.1) & 2 & {$[0.16$} \\
\hline Associated meniscal suture & 6 & $(18.75)$ & 3 & (9.4) & 2 & {$[0.42$} \\
\hline \multicolumn{7}{|l|}{ Gender (c) } \\
\hline Women & 3 & (9.4) & 7 & (21.9) & 0.43 & {$[0.09$} \\
\hline Man & 13 & $(40.6)$ & 9 & $(28.1)$ & 1.44 & {$[0.48$} \\
\hline
\end{tabular}

The statistical analysis of Table II reveals an ORb of 0.22 and a statistically significant difference $(\rho \square-$ value $<0.05$ ) for the control group as a whole.

The same is true for the 3-month subgroup with an ORb of 5.25 and the test group with an ORb of 4.45. However, the analysis presented in Table III shows no statistically significant difference for the sub group at 6 months for this group the OR b is 3.7. 
For all the variables where the statistical analysis shows no statistically significant difference, there is nevertheless an interaction between the variable and the risk.

Multivariate statistical analysis reveals statistically significant differences for BMI, age (over 35) and being

Indeed, we can read that the ORa for a normal BMI is 1.14 ; against 1.29 for a BMI which reflects overweight.

Likewise, the Odd Ratio is 1.67 for subjects over 35 years old and 1.44 for men.

Table I V : Results obtained for the test group within 6 months postoperatively.

\begin{tabular}{|c|c|c|c|}
\hline TDN & $95 \% \mathrm{Cl}$ & $\rho \square(b)$ & $\rho \square(b)(C M H)$ \\
\hline$(n=$ & & & \\
\hline not & & & \\
\hline
\end{tabular}

Age (c)

$\begin{array}{lllllllll}\begin{array}{l}\text { 18-25 years } \\ \text { 25-35 years }\end{array} & 3 & (9.4) & 3 & (9.4) & 1.42 & {[0.2431-8.2567]} & \text { NS } & \text { NS } \\ \begin{array}{l}\text { > } 35 \text { years old } \\ \text { BMI (c) }\end{array} & 5 & (15.6) & 9 & (28.1) & 0.79 & {[0.3103-3.0056]} & \text { NS } & \text { NS } \\ \begin{array}{l}18.5-24.99 \\ >24.99\end{array} & 3 & (12.5) & 5 & (15.6) & 1.13 & {[0.2508-5.1213]} & \text { NS } & \text { NS } \\ & 9 & (28.1) & 7 & (21.9) & 1.82 & {[0.5305-6.254]} & \text { NS } & \text { NS }\end{array}$

ACL reconstruction by DIDT ligamentoplasty (c)

\begin{tabular}{|c|c|c|c|c|c|c|c|c|}
\hline Isolated & 6 & (18.75) & 12 & (37.5) & 0.71 & [0.2075-2.4171] & NS & NS \\
\hline Associated meniscectomy & 0 & (0) & 2 & $(6.25)$ & / & / & NS & NS \\
\hline $\begin{array}{l}\text { Associated meniscal } \\
\text { suture }\end{array}$ & 6 & $(18.75)$ & 3 & $(9.4)$ & 2.83 & [0.5891-13.6276] & NS & 0.0166 \\
\hline \multicolumn{9}{|l|}{ Gender (c) } \\
\hline Women & 5 & $(15.6)$ & 7 & (21.9) & 1.01 & {$[0.2584-3.3623]$} & NS & NS \\
\hline Man & 7 & (21.9) & 10 & (31.25) & 0.99 & [0.2939-3.3461] & NS & NS \\
\hline
\end{tabular}

(a) adjustment for potential confounding factors : age, BMI, sex, associated lesions

(b) * NS = non-significant difference

(c) at the time of the CT scan or at the end of the survey for the control group

$\mathrm{CT}=$ trouble walking

$\mathrm{OR}=$ Odd Ratio

$(\mathrm{MH})=$ Cochran-Mantel-Haenszel $X^{2}$ test

$\varnothing \square=$ absence

For all the variables where the statistical analysis shows no statistically significant difference, there is nevertheless a link between the variable and the risk. 
The multivariate statistical analysis nevertheless reveals a statistically significant difference for the BMI when the subject is overweight.

Indeed, we can read that the ORa is worth 1.82 for a BMI which reflects overweight.

\section{DISCUSSION}

Results analysis

The study carried out will make it possible to compare walking disorders in patients operated on at 3 months and 6 months postoperatively. The analysis of the results according to age, BMI, sex and the associated surgical procedure makes it possible to assess the risk according to these criteria on the test group compared to the control group. The objective of this study is not to evaluate the influence of the operative technique at DIDT on the spatio-temporal parameters of walking. Indeed, the goal is to allow clinicians to obtain information about the walking disorders that can occur as well as the risk factors that cause them.

We can then say from the results of Table I that being asymptomatic is a protective factor with regard to the risk of having walking disorders, and this significantly.

In addition, it can be said that, also significantly, being operated on for reconstruction of the cruciate ligament anterior in the general case is an aggravating factor in terms of the risk of having walking disorders. Indeed, the $\mathrm{ORb}$ is 4.45 which allows us to confirm the trend.

As for the fact of being operated on for an ACL reconstruction by hamstring graft after 3 months of operation, we note that this is also an aggravating factor in terms of the risk of having a gait disorder. But we can say that it is even more so than in the test group because the ORb is equal to 5.25 which allows us to say that the general risk is higher, and this always significantly. The unified and multivariate analysis of the subjects of the test subgroup at the postoperative period of 3 months is summarized in Table III.

The univariate analysis reveals no statistically significant difference for the variables studied. Indeed, all the $\rho$-values for the Exact Fischer test are greater than 0.05 . There is therefore no link between the ORa and the variable studied.

However we can say wether there is a tendency to protect, to the aggravation or if there is no effect. From the results found in Table III, we can then say that there is a tendency to worsen the risk of having a gait disorder at 3 months postoperatively when the subject is older than age 35 ( ORa = 1.67), when the BMI indicates the subject's overweight ( ORa =1.29), when the subject has undergone an associated surgical procedure such as a meniscal suture or a meniscectomy $(\mathrm{ORa}=2)$ or finally when the subject is a man ( ORa $=1.44)$.

We will then determine if there are confounding factors for these variables, the confounding factors will be revealed by the Mantel-Haenszel adjustment method. 
Here, we can then say that subjects with a normal BMI (ORa $=1.14)$ have no more risk of having a gait disorder than the healthy population.

On the other hand, for this analysis, we can still consider in a non-significant way that the female subjects $(\mathrm{OR}=0.43)$, the subjects having undergone an isolated anterior cruciate ligament reconstruction ( $\mathrm{ORa}=0.67$ ) and the subjects whose the age is between 18 and 25 years ( ORa =0.67) or 25 and 35 years ( ORa $=0.5$ ) present a tendency to protect the risk of having a walking disorder in the post-period 3-month operation.

From Table IV, it seems that gender tends to no longer have an effect on the risk of gait disturbance at 6 months ( $\mathrm{ORa}=1.01$ for women and $\mathrm{ORa}=0,99$ for men), it is the same for subjects whose age is over 35 years ( $\mathrm{ORa}=1.13)$.

On the other hand, we can say that there is a tendency to protect the risk of having a gait disorder for the subgroups representing subjects between 25 and 35 years old ( ORa $=0.79$ ), subjects having a normal BMI ( ORa = 0.42); and subjects having undergone ACL reconstruction with isolated DIDT ( ORa = 0.71).

Nevertheless, it appears a tendency to worsen the risk of having a gait disorder when the subjects are aged between 18 and 25 years $(O R=1.42)$, when the subjects are overweight $(O R=1.82)$, and when there was an associated meniscal suture ( ORa $=2.83)$.

Here we can note that the $\rho$-value of the OR was equal to 1 for subjects between 18 and 25 years old, which reveals the non-incidence of age on the variable for this stratum.

The multivariate statistical analysis in Table IV enabled us, as for the previous group, to eliminate confounding factors if they exist.

The 6-month operating time test subgroup followed the same adjustment method as the 3-month group, the Cochran-Mantel-Haenszel test.

In this group, the statistical analysis revealed no difference for age, sex, subjects with a normal BMI and those who had an anterior cruciate ligament reconstruction in isolation.

We can therefore conclude that there is no interaction between the variables concerned and the gross risk expressed at 6 months (see Table II). Indeed, for these variables all $\rho(C M H)$ are greater than 0.05 . In addition, we note that the analysis shows a statistically significant difference for the variables concerning subjects with a BMI which reflects overweight ( $\rho=0.0348)$ and subjects having undergone a meniscal suture associated with ACL reconstruction ( $\rho=0.0166)$.

Regarding these last two variables, the Cochran-Mantel-Haenszel adjustment method therefore shows that there are interactions between the variable and the gross risk expressed at 6 months (see Table II). Here too, it turns out that these two interactions are confounding factors.

Thus, the risk of obtaining walking disorders after the postoperative period of 6 months amounts to the risk of ORa only for these two variables (see Table IV).

But the ORa is not significant, this allows us to say that at six months post-surgery, the risk is likely to reach the adjusted risk for these two variables. 
Sigward (19), tells us in a study that, clinically speaking, walking should normalize between 8 to 12 weeks after $\mathrm{ACL}$ reconstruction. However, many authors have reported walking disorders a few weeks after the operation, up to 12,13 sometimes even 24 months (20-23).

The results found in the literature are not necessarily identical because of the difference in postoperative time of the subjects included and the gait analysis protocol as well as the device used. Indeed, many studies are carried out with a 3D gait analysis device.

Di Stasi et al (15) were interested in adaptations of the gait depending on the sex of the preoperative phase where they undergo a rehabilitation program and up to 6 months after $A C L$ reconstruction.

At 6 months postoperative, the highlighted results show that the men present more asymmetry in their gait than the women. The study reveals a statistically significant value in men $(\rho<0.001)$, indicating that the hip range of motion of the operated limb was smaller than that of the unoperated limb, it is the same for its maximum knee flexion range when walking $(\rho<0.001)$. Asaeda et al in 2017 (14) demonstrated the presence of walking disorders 12 months postoperatively and that differences could still be noted between men and women.

The walking disorders identified are not the same but the two sexes present them in a similar way and make their walking non-physiological at 6 months and 12 months postoperatively. The study even concludes by arguing that it is not safe for women to resume sport at 6 months with such walking faults. Unlike them, in the present study there was no gait disturbance in women at 3 months and 6 months, however the postoperative delay of 12 months was not analyzed for our part. Few studies report data on the influence of age on walking disorders after ACL reconstruction. However, De Oliveira (16) shows in his 2019 study that there is a difference between young adults and adults.

Following our results, we notice that at 3 months it is the subjects over 35 years who present a worsening tendency to present a gait disorder and at 6 months it is the age group. age 18-25 years.

This analysis may lead us to think that older adults are cautious and more alert at the start of their rehabilitation and perhaps take more time in terms of functional recovery and that young people, on the contrary, give goodwill at the start of rehabilitation but that weariness settles gradually to measure.

Despite the scourge of overweight in the health field, there is no study that links BMI and gait disorders after ACL reconstruction. Nevertheless, Pietrosimone et al (10) in 2018 studied the associations between the body mass index and self-declared disability in people who had undergone unilateral reconstruction of the anterior cruciate ligament. The study is conducted through a questionnaire which will give a certain IKDC (International Knee Documentation Committee) score.

There are studies that analyze walking in overweight or obese subjects, but in healthy patients who have never undergone $\mathrm{ACL}$ reconstruction or any other damage to both lower limbs. Here, the literature agrees (24-26) to say that subjects with a BMI reflecting overweight or obesity present walking disorders both in terms of spatio-temporal benchmarks and qualitatively. Indeed, we notice adaptations of the above and underlying joints but also of the knee, slower walking, longer single and double support times, reduced joint moments. All these changes that occur in the walking pattern are not found in a healthy population whose BMI does not reflect overweight or obesity.

Analysis studies of walking with weighted vests simulating overweight were carried out by Hartigan et al $(27,28)$ respectively in 2017 and 2016, this time on operated patients. 
The persistence of hip asymmetries (only for women) and the moment of knee extension (for both sexes) are still apparent, and even worsened with the weighted vest.

These two studies tend to show that regardless of the operating time, overweight generates walking disorders after reconstruction of the anterior cruciate ligament. Although the subjects are not overweight in the last two, the weighted vest simulates overweight and thus approximates it.

In fact, it is recalled that in the present study, the patients present gait disorders associated with a BMI reflecting overweight at 3 months and at 6 months postoperatively, which is in agreement with the literature.

However, it is important to note that the BMI is not always revealing because many athletes have a BMI which reflects being overweight simply because the muscle mass is heavier than the fat mass.

A few authors have been interested in walking disorders and alterations in the biomechanics of the knee after $\mathrm{ACL}$ reconstruction by hamstring graft associated with a meniscal lesion (partial meniscectomy or meniscal suture).

In 2016, Hall et al (9) are interested in meniscal injury and seek to know if the latter modifies the biomechanics of gait and the strength of the knee after ACL reconstruction with DIDT. In this study, the subjects have all been operated for 12 to 24 months and the associated meniscal lesions are only partial meniscectomies or meniscal sutures.

The results of the study do not allow us to demonstrate that the meniscal lesion associated with $\mathrm{ACL}$ reconstruction with hamstring graft is responsible for a gait disorder or a lack of strength in comparison with a population operated on for reconstruction of the $A C L$ in isolation.

Capin et al (12), in 2018 published a study following on from Hall et al's work on changes in the biomechanics of gait after ACL reconstruction associated with a lesion of the medial meniscus. In this study, the subjects participating in the study have had surgery for an average of 5.3 months.

The results of the study show that there is a link between the alteration of the spatio-temporal landmarks of walking and the meniscal suture (here medial) in comparison with a healthy population. Other information comes to the fore when Capin compares the group that received a partial meniscectomy with the group that received a meniscal suture. In fact, he notices that the subjects of the partial meniscectomy group load the operated limb on the medial compartment of the knee while the subjects of the suture group tend to load the contralateral, non-operated limb.

In 2019, Capin et al (13) are interested this time in the consequences that may have on the biomechanics of walking in subjects who have had a partial meniscectomy or a suture two years after ACL reconstruction.

The results of the study show that the walking pattern is altered within two years after reconstruction associated with partial meniscectomy but not in the case of a suture associated with ACL reconstruction. The analysis of the results of this study, which takes place on subjects with a postoperative delay of 2 years, seems to highlight the fact that meniscectomy would be more harmful in the long term than meniscal suturing. Indeed, one can suppose that the absence of meniscus which alters the stability of the knee, modifies the application of the constraints and thus could lead to unwanted cartilaginous lesions which would come to contribute to the alteration of our walking pattern.

From another point of view, Asaeda (14) who studied walking defaults depending on gender comes to the conclusion that, according to the current database, the differences between the sexes in the 
occurrence of post-operatory osteoarthritis probably comes most likely from the occurrence of meniscal and ligament injuries instead of the specific difference in gender in the biomechanics of walking.

The results put forward by Capin $(12,13)$ and his collaborators in 2018 are in agreement with the results of the statistical analysis in the present study. Indeed, the postoperative delay is the closest to our study with 5.3 months and they also find walking disorders in subjects who have benefited from an associated meniscal suture.

\section{Limits}

First of all, it is important to report the number of participants within each stratum and we know that the size of the samples has an influence on the significance of the results obtained. We can then wonder if these results could have been modulated with a larger sample.

Thus, we can in no way affirm that the results of the present study are representative for a larger sample. First, it would be interesting to increase the number of subjects in the control group in order to balance each group optimally.

It would perhaps be advisable to carry out the study again while keeping the same selection criteria to maintain the same objective by increasing the cohorts of subjects to allow better stratification and thus a more efficient analysis of the results.

Today there is no real consensus in the exercises that can be offered in the context of rehabilitation following $\mathrm{ACL}$ reconstruction by hamstring graft.

All the subjects from the LCA group were operated on in the same clinic with three different surgeons but according to a common protocol. The post-operative surgical instructions which were given for the rehabilitation follow-up are specified and are the same for all subjects. This limits the variability associated with several therapists even if they were then followed by different physiotherapists in their practice and therefore did not all follow the same rehabilitation program.

In a study published very recently; Gokeler, Vascellari et al (29) question the time delays to which we are often subjected throughout $A C L$ rehabilitation. The results of their study, performed primarily by orthopedic surgeons, indicate that functional landmarks are more appropriate than steps based on time since operation to guide progression in post-operative rehabilitation after ACL reconstruction. Thus they describe " phases " of rehabilitation: an early phase, an intermediate phase and a phase of return to sport. To move from one phase to another, joint, muscular, neuromuscular and / or functional objectives are expected. The author does not specify whether walking is a criterion for moving to the next level.

As for walking, there is no specific recommendation in the literature to carry out its rehabilitation. However, each physiotherapist is completely free to use the techniques that seem optimal, effective and necessary during rehabilitation in order to achieve his goals. In their study, Shi et al (3) show that treadmill walking can lead to a reduction in stride length and duration. On the other hand, it seems that it can increase the stability of the gait of the upper body but decrease the regularity of the gait during the swing phase. 
One possible interpretation of these results would be that people tend to walk more cautiously to avoid the risk of falling and, as a result, neglect the coordination between gait and upper trunk when walking on the treadmill.

Indeed, in many studies carried out in the literature, the analysis of walking is done on the ground $(12,15)$ or on a force platform $(13,27,28)$, over a given distance $(15)$ or not and at a speed determined by the subject $(12,13,15,27,28)$.

Furthermore, subjects from the $A C L$ group were all operated using a reconstruction by hamstring graft. Indeed, one can wonder if the surgery used could influence the appearance of walking disorders in the postoperative phase. In the literature, most studies seem to agree that there is no significant difference between reconstruction with hamstring graft or with the Kenneth Jones method.

In a study published in 2019, Johnston reports that there is no difference between the two operating modes with respect to the spatio-temporal parameters of walking and that neither would seem to favor the early onset of acquired osteoarthritis in the postoperative phase (30).

Nevertheless, the biases presented remain however inherent in this type of research for the most part and are acceptable. Indeed, in view of the results and conclusions which remain in agreement with the literature, this remains relevant in the context studied.

\section{CONCLUSION}

The main objective of the study was to determine what are the risk factors for gait disturbance following $\mathrm{ACL}$ reconstruction by hamstring graft at 3 months and 6 months postoperatively. A non-randomized study was therefore carried out by comparing patients who presented walking disorders at 3 months with a healthy population, and patients who presented walking disorders at 6 months with this same healthy population. The comparisons were made according to variables which are identical in the two subgroups of the test group. Namely, the study compared the walking disorders according to the sex, the age, the BMI of the subjects and finally the presence of a surgical procedure associated or not to the reconstruction of the $A C L$.

Analysis of the results of subjects with a postoperative period of 3 months highlights many trends.

The altered gait is found in each stratum, however the method highlights tendencies to worsen the risk of obtaining a gait disorder in males, subjects who have benefited from an associated surgical procedure, subjects over 35 years of age and those with a BMI indicating overweight. An absence of statistically significant difference is found during the analysis for the other variables, which means that the variables in question have no effect on the risk of obtaining gait disturbances at 3 months postoperatively.

On the other hand, we note that at 6 months, the analysis of the results reveals a tendency to worsen the risk of obtaining a gait disorder only in subjects who have a BMI reflecting overweight those having benefited from a meniscal suture.

An absence of statistically significant difference is found during the analysis for the other variables, which can testify that the variables in question have no effect on the risk of obtaining gait disturbances at 6 months postoperatively. 
The analysis of gait in subjects having benefited from $A C L$ reconstruction by hamstring graft tends to show an alteration of the spatio-temporal parameters of long-term gait in patients with a BMI reflecting overweight and in subjects who have had an associated meniscal injury. Thus, it would be interesting to adapt our management in order to re-educate as well as possible these patients who are more at risk of getting walking disorders than others.

In addition, the integration of an analysis of gait associated with a muscle evaluation, posture and laxity of the transplant at different stages of rehabilitation would make it possible to adapt the treatment with a view to sports recovery and avoid the onset of complications or recurrence.

Furthermore, the use of tools such as OptoGait ; allowing us to analyze quantitatively walking and spatial and temporal parameters, should be seen as a usefull device in rehabilitation and could be implemented in such protocols.

The analysis of walking, whether qualitative or quantitative, tends to show deficits that can persist for several years after $\mathrm{ACL}$ reconstruction. The rupture of the ACL targeting mainly a sports public, and in view of the new found interest in running, it is crucial to evaluate the walk in all its components for rehabilitation, as well as possible to prevent the secondary lesions which can occur following a poor walking regimen and running. All this obviously with the aim of improving the quality of the assessment and therefore the rehabilitative care of these patients.

1. Majewski M, Susanne H, Klaus S. Epidemiology of athletic knee injuries: A 10-year study. The Knee . 2006 Jun ; 13 (3): 184-8.

2. Fuentes A. Contribution of a 3D biomechanical evaluation of the knee in the orthopedic management of patients with an anterior cruciate ligament rupture. [Montreal ]: University of Montreal Faculty of Graduate and Postdoctoral Studies; 2010.

3. Shi L, Duan F, Yang Y, Sun Z. The Effect of Treadmill Walking on Gait and Upper Trunk through Linear and Nonlinear Analysis Methods. Sensors. 2019 May 13 ; 19 (9): 2204.

4. Nebelung W, Wuschech H. Thirty-five Years of Follow-up of Anterior Cruciate Ligament Deficient Knees in High-Level Athletes. Arthrosc J Arthrosc Relat Surg. 2005 Jun ; 21 (6): 696702.

5. Naranje S, Mittal R, Nag H, Sharma R. Arthroscopic and magnetic resonance imaging evaluation of meniscus lesions in the chronic anterior cruciate ligament-deficient knee. Arthroscopy . 24 (9): 104551.

6. Trojani C, Remi M, Neyret P, Boileau P. Natural history of the knee after rupture of the anterior cruciate ligament in adults. SOFCOT teaching notebooks $\mathrm{N}^{\circ}$ 86, p 8-16. 2004.

7. Kennedy J, Jackson MP, O'Kelly P, Moran R. Timing of reconstruction of the anterior cruciate ligament in athletes and the incidence of secondary pathology within the knee. J Bone Joint Surg Br. 2010 Mar; 92- B ( 3): 362-6.

8. Joseph AM, Collins CL, Henke NM, Yard EE, Fields SK, Comstock RD. A Multisport Epidemiologic Comparison of Anterior Cruciate Ligament Injuries in High School Athletics. J Athl Train. 2013 Dec ; 48 (6): 810-7.

9. Hall M, Bryant AL, Wrigley TV, Pratt C, Crossley KM, Whitehead TS, et al. Does meniscal pathology alter gait knee biomechanics and strength post-ACL reconstruction? Knee Surg Sports Traumatol Arthrosc . 2016 May ; 24 (5): 1501-9.

10. Pietrosimone B, Seeley MK, Johnston C, Pfeiffer SJ, Spang JT, Blackburn JT. Walking Ground Reaction Force Post-ACL Reconstruction: Analysis of Time and Symptoms. Med Sci Sports Exerc . 2019 Feb ; 51 (2): 246-54.

11. Capin JJ, Zarzycki R, Arundale A, Cummer K, Snyder- Mackler L. Report of the Primary Outcomes for Gait Mechanics in Men of the ACL-SPORTS Trial: Secondary Prevention With and 
Without Perturbation Training Does Not Restore Gait Symmetry in Men 1 or 2 Years After ACL Reconstruction: Clin Orthop. 2017 Oct; 475 (10): 2513-22.

12. Capin JJ, Khandha A, Zarzycki R, Manal K, Buchanan TS, Snyder- Mackler L. Gait Mechanics After ACL Reconstruction Differ According to Medial Meniscal Treatment: J Bone Jt Surg. 2018 Jul ; 100 (14): 1209-16.

13. Capin JJ, Khandha A, Buchanan TS, Snyder- Mackler L. Partial medial meniscectomy leads to altered walking mechanics two years after anterior cruciate ligament reconstruction: Meniscal repair does not. Gait Posture. Oct 2019; 74: 87 -93.

14. Asaeda M, Deie M, Fujita N, Kono Y, Terai C, Kuwahara W, et al. Gender differences in the restoration of knee joint biomechanics during gait after anterior cruciate ligament reconstruction. The Knee. 2017 Mar; 24 (2): 280-8.

15. Di Stasi S, Hartigan EH, Snyder- Mackler L. Sex-Specific Gait Adaptations Prior to and up to 6 Months After Anterior Cruciate Ligament Reconstruction. J Orthop Sports Phys Ther . 2015 Mar; 45 (3): 207-14.

16. de Oliveira EA, Andrade AO, Vieira MF. Linear and nonlinear measures of gait variability after anterior cruciate ligament reconstruction. J Electromyogr Kinesiol . Jun 2019; 46: 21 -7.

17. Majewska J, Szczepanik M, Szymczyk D, Bazarnik-Mucha K, Drużbicki M, Snela S, et al. Evaluation of certain parameters of the patient's gait before and 6 months after the intervention of early reconstruction of the anterior cruciate ligament. : 11 .

18. Shin S. Agreement between the spatio -temporal gait parameters from treadmill-based photoelectric cell and the instrumented treadmill system in healthy young adults and stroke patients. Med Sci Monit . 2014; 20: 1210-9.

19. Sigward SM, Chan M-SM, Lin PE. Characterizing knee loading asymmetry in individuals following anterior cruciate ligament reconstruction using inertial sensors. Gait Posture. 2016 Sep ; 49: 114-9.

20. Shi H, Huang H, Ren S, Yu Y, Liang Z, Wang Q, et al. The relationship between quadriceps strength asymmetry and knee biomechanics asymmetry during walking in individuals with anterior cruciate ligament reconstruction. Gait Posture. Sep 2019; 73: 74-9.

21. Robbins SMK, Clark JM, Maly MR. Longitudinal Gait and Strength Changes Prior to and Following an Anterior Cruciate Ligament Rupture and Surgical Reconstruction: A Case Report. J Orthop Sports Phys Ther . 2011 Mar; 41 (3): 191-B4.

22. Slater LV, Hart JM, Kelly AR, Kuenze CM. Progressive Changes in Walking Kinematics and Kinetics After Anterior Cruciate Ligament Injury and Reconstruction: A Review and MetaAnalysis. J Athl Train. 2017 Sep; 52 (9): 847-60.

23. Davis-Wilson HC, Pfeiffer SJ, Johnston CD, Seeley MK, Harkey MS, Blackburn JT, et al. Bilateral Gait 6 and 12 Months Post - Anterior Cruciate Ligament Reconstruction Compared with Controls: Med Sci Sports Exerc . 2020 Apr ; 52 (4): 785-94.

24. Lai PPK, Leung AKL, Li ANM, Zhang M. Three-dimensional gait analysis of obese adults. Clin Biomech . Jan 2008; 23: S 2-6.

25. Runhaar J, Koes BW, Clockaerts S, Bierma -Zeinstra SMA. A systematic review on changed biomechanics of lower extremities in obese individuals: a possible role in development of osteoarthritis: Obese biomechanics of everyday movements. Obes Rev. 2011 Dec ; 12 (12): 107182.

26. Rosso V, Agostini V, Takeda R, Tadano S, Gastaldi L. Influence of BMI on Gait Characteristics of Young Adults: 3D Evaluation Using Inertial Sensors. Sensors. 2019 Sep 28; 19 (19): 4221.

27. Hartigan E, Aucoin J, Carlson R, Klieber-Kusak M, Murray T, Shaw B, et al. Relationships

Between Knee Extension Moments During Weighted and Unweighted Gait and Strength Measures

That Predict Knee Moments After ACL Reconstruction. Sports

Health Multidiscip Approach. 2017 Jul ; 9 (4): 356-63.

28. Hartigan E, Lawrence M, Murray T, Shaw B, Collins E, Powers K, et al. Biomechanical Profiles When Towing a Sled and Wearing a Weighted Vest Once Cleared for Sports Post - ACL

Reconstruction. Sports Health Multidiscip Approach. 2016 Sep; 8 (5): 456-64.

29. Vascellari A, Gokeler A, Grassi A, Canata GL, Zaffagnini S, Jones H. Functional progression milestones following anterior cruciate ligament reconstruction are more appropriate than time-based 
criteria: a survey among the ESSKA. Knee Surg Sports Traumatol Arthrosc [Internet]. 2 Apr 2020 [cited 11 Apr 2020 ]; Available at: http://link.springer.com/10.1007/s00167-020-05960-3 30. Johnston CD, Goodwin JS, Spang JT, Pietrosimone B, Blackburn JT. Gait biomechanics in individuals with patellar tendon and hamstring tendon anterior cruciate ligament reconstruction grafts. J Biomech . Jan 2019; 82: 103-8. 